\section{Beiträge zur Kenntniss der wandernden Pneumonie.}

\section{Von}

\section{Dr. B. Kuessner in Halle.}

(Schluss aus No. 7.)

Was den zuletzt mitgetheilten Fall betrifft, so kann kein Zweifel daräber sein, dass es sich anch hier um eine wanderade croupöse Pnenmonie gehandelt hat - die dentlichsten physikalischen Zeichen einer Infiltration, welche an einer Stelle vollkonımen rückgäıgig wird, um zur andern vorznschreiten, der gntartige nnd relativ kurzdanernde Verlant (wenigstens hei jedem einzeluen Infiltrat) lassen etwas Anderes kanm ınöglich erscheinen; wir kennen gar keine andern Infiltrationsznstände mit solchem Verlanf: eine „katarrhalische“" Pueumonie ist eo ipso anszuschliessen, nnd anch von phthisischen oder tnberknlösen Verdichtungen kann gar keine Rede sein. Im höchsten Grade anffallend nod darnm Anfangs die Diagnose so sehr erschwerend, musste natürlich das Spntum sein: während der ganzen Daner der Krankheit nicht ein einziges Mal rostfarbiger Answurf - das ist fast nuerhört bei einem jugendlichen Individnnm!

Das ferruginöse Sputnm wird so gut wie allgemein als ein HanptCharakteristicum der croupöse a Puenmonie angesehen, ja eine solche wird öfters ans dem Sputum allein diagnostizirt ${ }^{1}$ ), anch wenn sie sonst latent, d. h. phlysikalisch nicht nachweisbar verlänft. ("Centrale Pneumonie", wovon ein Beispiel weiter unten angefülırt werden soll.) Es kann wohl passiren, dass bei einer Pnenmonie gar kein Answurf vorhanden ist - bekanutlich legten die älteren Aerzte Gewicltt daranf, dass überhanpt expectorirt wird, weil sonst eine Puenmonie nicht den normalen Verlanf dnrchmachıen könne, sich nicht ordentlich resolvire etc.; wenn wir diese Meinnng anch hente nicht in vollem Unufange theilen, so müssen wir doch zngestehen, dass ein gewisses Maass von Answurf zn den "normalen" Erscheinnngen der Pnenmonie gehört, und anch prognostisch günstig ist; natürlich immer voransgesetzt, dass der Kranke nicht, wie Kinder thnn, das Sputnm verschluckt, dass also Sputnm überhanpt secernirt wird. Wo dies der Fall ist, nnd zwar wo die erkrankten Theile der Lnnge das Spntnm liefern, da enthält es, wenigstens bei jüngeren Leuten, Blnt in der eigenthümlichen rostfarbigen Beimischnng. Es ist bekannt, dass bei alten Lenten, besonders wenn sie schwach sind und an langwierigen Katarrhen leiden, der Answarf nicht selten rein schleimig-eitrig ist, sowie anch dass Aehuliches beohachtet wird hei jüngeren Individnen, die mit chronischen Katarrhen (Emphysem u.s. w.) behaftet sind, nnd dass eben desswegen die Diagnose in solchen Fällen nicht selten anf Schwierigkeiten stösst. Schon Andral (Clin. méd. I.) hat auf diese Eigenthümlichkeiten des Sputnıs mit aller Schärfe hingewiesen. Im Uehrigen scheint aber, wie erwälınt, ein wenn anch geringer Blntgehalt der Sputa so gat wie allgemein als conditio sine qua non zn gelten; ich habe mich grade über diesen Pankt hesonders genan zu orientiren gesncht. Grisolle (Traité prat. da la puenmonie) allerdings sahı in 20 Fällen [über deren Alter ete. freilich nichts Näheres angegeben ist] während der ganzen Dauer der Krankheit unr weissliches, katarrhalisches Spntnm. Und bei M. Huss (Lungenentzündung, ühersetzt von Anger) heisst es: „Mitunter ist während der ganzen Daner der Krankheit das Spntım katarrialisch, die ersten 3-5 Tage farblos, später opalisirend nnd perlfarbig. Dies ist der Fall bei gewissen Langenentzündungen, die sich ans einer capillären Bronchitis entwickeln." 0 b es sich aber in diesen Fällen nm richtige croupöse Pnenmonie handelte? Alle übrigen Autoren betrachten einen Blntgehalt des Spntums so zu sagen als selbst verständlich.

Jedenfalls ist bei unserm Kranken, einem jungen soust kräftigen Menschen ohne Katarrh, das Spntnm znmal bei seiner Reichlichkeit nahezu ein Unicam. Nor in einer Beohachtung habe ich etwas Aehnliches gefnnden; dieselhe rührt von Fischel her und betrifft ebenfalls eine Wanderpueumouie (Prager Vierteljahrsschrift 1872). Es handelte sich um eine 36 jährige, kräftige Fran, deren Krankheit 6 Wochen dauerte und mit Genesnng endete; die einzelnen Infiltrate resorhirten sich immer in wenigen Tagen. Es waren nur schleimig-eitrige Sputa

1) Sehr gründlich ist diese Frage z. B. von Eich horst (Handb. d. spec. Path. u. Ther. I.) ganz in dem erwähnten Sinne behandelt. vorhanden. - In einigen der von Homhnrger (l.c.) mitgetheilten Fälle wnrde zeitweise nnr gelbes oder grünlich-gelbes Sputnm hinansbefördert, das hald zäh bald locker war; im Uehrigen fehlte aber das charakteristische ferruginöse Sputum nicht. Anch Znber (Art. „Crachats“ im Dict. elıcycl. des sc. méd. par M. Decha nhre) erwähnt in seiner löchst eingehenden nnd sorgfältigen Darstellnng nichts derartiges.

Werfen wir noch einige Blicke anf gewisse Einzelheiten, die grade mit Räcksicht anf das Verhältniss der Wanderpuenmonie zu deu „einfachen“ Puenmonien von Interesse sind. Die Hauptfrage in rein practischer Beziehnng, ob die Wanderpuenmonie sich durch grössere Maligniläı anszeichnet nnd demgeınäss eine schlechtere Prognose giebt oder nicht, wird von vielen Antoren nicht erwähnt. Friedreich (Volkmann's Samml. klin. Vortr. No. 75), der sie anch zn einer besonderen Art stempeln will, erklärt sie für eine prognostich nugüustige Erkrankung; ebenso lıält Tronssean (Clitı. méd. I.) sie für vorzngsweise schlimm. Zieınssen (Plenritis nnd Pneumonie im Kindesalter) dagegen sah, wenigstens bei Kindern, wandernde („saccadirte“) Pnenmonien mit gntartigeın Verlanf; er hält dabei vorhandene resp. forthestehende febrile Temperaturen für günstig, wie wir ja diese Erscheinung anch hei der einfachen croupösen Puenınonie z.n den ,normalen" and darum güıstigeı rechnen.

Es stehen sich also hinsichtlich dieses Punktes Namen von gewichtigstem Klange in ihren Anschanungen gegenüber. Hombnrger, der in dieser Beziehnng ohne Zweifel anch K us smaul's Anschannngen wiedergiebt, erklärt die Puenmonia nigrans für nicht schlimıner als die P. crouposa siuplex.

Strü mpell (Lehrbnclı d. spec. Path. I.) sagt: „Die Wanderpneumonien sind fast immer schwere nnd relativ lange danernde Pnenmonien," nnd nusere 3 Fälle stimmen völlig damit überein - es sind schwere aber nicht besonders "maligne" Erkranknngen.

Wären es in Fall I nud II ,einfache" cronpöse Puenınonien gewesen, die tödtlich endeten - kein Mensch hälte sich wadern können! -

Sieht man die oben mitgetheilten Krankengeschichten etwas genaner an, so wird man finden, dass der Verlanf der einzelnen Infiltrate nicht imnıer derselbe ist - manche danerten nur wenige Tage, manche hielten sich Wochen lang. Ob bei den ersteren nicht der ganze "typische“ Process vom Engonement, zur rothen und granen Hepatisation etc. sich abspielte, lässt sich nach der klinischen Beobachttnng natürlich nicht sagen - es ist ja aber bekannt, dass anch die „einfache“ cronpöse P. mitnnter sehr capriciös in dieser Beziehnng ist. Es sind ja selbst eintägige Pnenmonien beobachtet (Weil, Lenbe, Bernhardt), wo von eineun cyklischen Verlauf füglich nicht die Rede sein kann. Wahrscheinlich spielt die Individualität hier eine grosse Rolle. Waldeuhurg (l. c.) fand, dass die einzelnen Infiltrate nur abortiv verliefen; Wnnderlich giebt an, dass dieselben bei der Pnenmonia migrans im Allgemeinen einen raschen Verlanf zeigen, nnd Homburger hat das bestätigt gefunden; nusere Fälle zeigen, dass auch dies sehr verschieden sein kann.

Es ist an dieser Stelle nöthig darauf hinzuweisen, dass anch bei den gewöhnlichen croupösen Pueumonien die zeillichen Verhältaisse der Infiltration dnrchans nicht immer dem Schema gemäss verlanfen, und zwar speciell dass eine unehr oder minder verzögerte Resolution hei schliesslichıen Ansgang in völlige Heilnng stattinden kann.

Koranyi (in Enlenburg's Real-Encyclop.) scheint zwar dies zu bezweifeln; wenigstens sagt er I. c.: ,oh eine gennine Puenmonie üher drei Wochen dauern kann, ist nicht ganz sichergestellt;" indessen erklären die meisten Autoren eine Verzögerung der Resolntion für nicht gar so selten (nnd zwar wohlbemerkt anch bei sonst ganz gesnnden Leuten); dass hei Emphysematikern, bei Säufern, hei Nephritikern, bei anderweitig geschwächten Lenten eine Pnenmonie viel langsamer verlanfen kann, ist eine allgemein anerkannte Thatsache. Einige haben auch versucht, eine Erklärung dafür zn geben. Eichhorst (l. c.) hat „protrahirte Pnenmonien“ von vier bis sechs Wochen Daner gesehen. Nach Lépine, dessen Artikel „Pnenmonie“ im Nouv. Dict. de méd. et chir. Bd. 28 (1880) zn dem Besten zählt, was über Pneumonie geschriehen worden ist, gehört verzögerte Resolution eines puenmonischen Infiltrats keineswegs zn den Seltenleiten und ist hesonders den französisclıen Antoren wohl bekanut. Anch Balzer (ibid,) ist derselhen Meinung, giebt an, dass selbst viele Wochen bis zn vollendeter Resolntion vergehen können, nad reprodncirt Charcot's Lehre, die folgendermaassen lantet: "Ces exsndats non résorliès persistent ainsi an sein du parenchyme pulmonaire, sans qu'il $y$ ait coëxistence d'un travail inflammatoire." Lebert (Klinik der Brnstkrankheiten) findet ehtenfalls, dass protrahirter Verlanf der Puenmonie uicht allzu selten ist, nnd zwar nicht blos bei Sänfern und anderweitig geschwächten Personen, sondern anch hei sonst gesnnden, in günstigen Allgemeinverhältnissen. Anch Eichh orst nud St rümpell [1l. cc.]) erwähnen derartige Vorkominnisse speciell and beschreiben sie genan. Besonders eingehend behandelte E. Wagner (D. Arch. f. klin. Med. 33) diesen practisch so ansserordentlich wich- 
tigen Gegenstand; and es wäre sehr zn wänschen, dass wir ähnlich sorgfältige Beobachtungen in grösserem Umfange besässen. Wir erwähnen endlich Leyden (Berl. klin. Wocheuschrift 1879), der ebenfalls äusserst instrnctive Beiträge zn dieser Frage geliefert nnd zugleich eine Erklärṇng zn geben versncht hat. Er maclit daranf anfmerksam, dass nicht blos bei alten, sondern anch bei ganz jungen Lenten verzögerte Resolution stattfinden kanu, nod schuldigt vorwiegend die Derbheit des Infiltrates an. Das ist sehr plansibel und ohne Zweifel für viele Fälle richtig, stimmt abcr wahrscheinlich nicht für alle. Wir machen wicderholt anf die oben angeführte Thatsache aufinerksam, dass gerade bei constitntionellen Anonıalien, Morbns Brightii, Alkoholisnıns etc. vorwiegend oft die Resolution eine langsamere als gewöhnliche ist, nud dass wir bei diesen Processen (wie anch anatonisch festgestellt) weit eher die "schlaffen" Infiltrate finden. Leyden weist ferner anf die gesunkene Circulation hin, ein Moment, das anch von Leichtenste rn für die Erklärnng der asthenischen Pnenmonien unit ihrer schlaffen Hepatisation hermgezogen wird. Nach Strünıpell (l. c.) sind bei Puenmonien von Emphysematikern die Alveolen nicht ganz durch das Exsudat ansgefüllt, and doch die langsame Resolntion!

Von Ueberbleibseln des paenmonischen Processes fand sich bei nnsern Kranken nur in Fall 1 eine mässige Einziehnng im Bereich der Spitze, als Ansdruck einer leichten Schrumpfnng. Es ist anch diese Frage (d. h. ob Schrnmpfnngszustände als Ansgangsprocesse der Puenmonie vorkommen) noch uicht als definitiv erledigt anzusehen. Jürgenseu erklärt interstitielle Processe nach croupöser Pnenmonie für nnzweifelhaft, aber nicht hänfig vorkomnıend; nach Balzer entwickelt sich Schrnmpfnıg öfters, entweder in einem Znge oder in Schäben; Lebert sah nach „chronischer Resolntion“ hänfig Ansgang in Sthrnmpfung. Dagegen liält Grisolle (l. c.) und ebenso nenerdings E. Wa g n er (l. c.) den Znsammenhang derselben mit echter primärer Pnenmonie für zweifelhaft, anch gegenüber den Befnnden von Marchand (Virchow's Archiv Bd. 82).

Ohne anf die anderen nach cronpöser Pnenmonie beobachteten Erkrankungen einzogehen, die anch bei Wagner besonders gründliche Würdignng gefunden haben, möchte ich nur noch die Anschannngen von M. Hnss knrz anführen, die als Resnltate langjähriger Stndien an einem grossen nnd eigenartigen Material gewiss volle Beachtnng verdienen. H. findet, dass Sänfer-Pueumonie häufiger als sonst Pnenmonie „chronisch“ wird (ohne tuberculös zn werden), [ob damit interstitielle Processe gemeint sind?] and dass Neigung zo zuräckbleibender "Verdiclıtnng“ der Lange niclıt selten anch bei beschränktem Umfange nud "gelinder Besclaffenheit der Entzäudang" besteht. (NB. Letzteres gilt nicht blos von der Säufer-Pnenmonie.)

An das soeben dargelegte möchte ich nur noch ein paar ganz kurze Bemerkungen unehr negativer Natnr knüpfen. ln keinem nuserer Fälle bestand ein typhöser Allgemeinzusland, wie ihn Friedreich in seinen Fällen so clarakteristisch fand; es war ferner kein Milztnmor zn constatiren, selbst in Fall II nur nnsicher - also volle Uebereinstimmnng niit den Angaben von Homburger (l. c.). Anch von Erysipelas, weder von einem gleichzeitigen, noch von einem vorher dagewesenen, war in keinem nuserer Fälle die Rede, und wenn wir anch für die Pueumonia migraus im Allgemeinen zngeben wollen, dass ihre Ausbreitung an die eines Erysipelas sehr lebhaft erinnern kann, so glauben wir doch, dass es falsch ist, darans anf eine uahe Verwandtschaft oder gar Identität beider Processe zn schliessen, wie dies mehr oder uninder direct von Waldeubarg, Friedreich (ll. cc.) nad Znelzer (Ziemsse n's Handbuclı II, 2) gescheheu ist; dass es vielmehr richtig ist, was Strämpell sagt: es habe diese rein änsserliche Aehılichkeit zn vielerlei verkehrten Vorstellnngen Anlass gegeben.

Als Anhang theile ich einen Fall von "centraler Pnenmonie“ mit, der nnr dazn dienen naag, die Kasnistik in dieser Beziehnng zn erweitern; schriftliche Notizen äber andere Fälle der Art, die mir mit aller Bestinmtheit vorgekommen sind, besitze ich leider nicht mehr. Ich glanbe aber, dass die meisten kiliniker eine centrale, d. h. physikalisch nicht nachweishare Pneumonie anerkennen werden; nnr Lépine (l. c.) drückt seine $\mathrm{Zweifel} \mathrm{daräber} \mathrm{ans.}$

Der Fall betrifft einen 28jälırigen Arbeiter J., der am 25. November 1878 in die medicinische Klinik hierselbst anfgenommen und am 23. December 1878 entlassen wurde.

Er war früher stets gesnnd gewesen, erkrankte am 21. November Abends mit heftigem Schüttelfrost, nachfolgender Hitze, Stechen in der linken Seite, Husten nod Kurzathmigkeit, schlief in der Nacht schlecht und expectorirtc vom nächsten Tage ab ein zähes, mit etwas Blut untermischtes Spntnm. Bei seiner Anfnahme konnte folgender Status notirt werden.

Kräftig gebauter Mann, Hant etwas fencht, Lippen nnd Wangen leicht cyanotisch gefärbt. Kein Herpes, 42 Respiratiouen in der Minute; die linke Thıraxhälfte bewegt sich etwas schwächer als die rechte.
Temperatnr öber $40^{\circ}$; im anffallenden Gegensatz dazn die Pulsfrequenz: 76 in der Minute! Die einzelnen Pnlse voll nnd weich. - Die Percussion ergiebt dnrchweg normale Verhältnisse, bei der Auscultation hört man überall vesicnläres Athmen, hinten liuks nuten bei tiefen Inspirationen znweilen nnbestimmtes Rasselı. Patient lınstet in mässiger Menge ein zähes, z. Th. rostfarbenes Spntnm ans. Leichte Albnminnrie. Sonst absolnt nichts Alınormes. Sensorinm völlig frei. Patient klagt uur üher die Stiche in der linken Seite. Bis znm 28. Noventher blieb der Zustand des Kranken ganz derselbe, namentlich der Befund äber den Lungen negativ; das Seitenstechen hielt, wenn anch etwas abuehmend, bis zn diesenn Tage an. Plenritisches Reiben war nicht zn hören. In Lanfe des 28. Noveınber fiel die bis dahin sehr hohe Temperatnr anf $37^{\circ}$ unter starkeı Scliweiss. Die anffallend niedrige Pulsfreqnenz (ca. 70) ging noch weiter hernnter, anf 44, selbst 40 Schläge nnd blieb 7 Tage lang so (Pat. hatte zn keiner Zeit Digitalis erhalten, unr Sol. Natr. nitric. 5-200 2stündlich 1 Esslöffel), hob sich daun ein wenig nnd schwankte ron da ab bis zn seiner Entlassung zwischen 50 nnd 60. Der Urin war nach der Krise danernd eiweissfrei.

Patient war bereits anfgestanden nnd fühlte sich, abgesehen von etwas Seitenstechen, ganz wohl bis zun 7. December, an diesen Tage trat leichte Temperatnrerhölıng $\left(38^{\circ}\right)$ ein, die Schmerzen wurden etwas stärker, so dass Pat. sich wieder zn Bett legte, nnd 8 Tage lang blieben die Beschwerden vermehrt, wenn anch imnierhin in sehr bescheidenen Grenzen, ebenso die Temperatnrsteigernng. Der plyssikalische Befund blieb danernd so gut wie negativ - links hinten unten nach Hustenstössen nnd bei ganz tiefen Inspirationen spärliches, dumpfes Rasseln, welches rechts felilte. Anch anf der linken Seite verlor es sich, so dass Pat. am 23. December geheilt entlassen werden konnte. Vielleicht ist also dieser Fall nnter die recidivirenden Pnenmonien zu rechnen. Leider vermag ich nicht mehr zn sagen, wie es in deın eben mitgetheilten Falle mit der Brouchophonie stand - die mir vorliegende krankengeschichte ist etwas fragnentarisch, und ich weiss uicht, wie weit ich meinem Gedächtnisse trauen darf. Ich möchte aber diese Gelegenheit benntzen, um grade das Verhalten der Broncliophonie nach abgelaufener Paeumouie zu erwähnen. Die Angaben der Antoren sind keineswegs äbereinstinmend; bei Vielen findet sich gar aichı darüber.

Bekannt ist es. dass oft lange Zeit nach der Krise nicht blos verschärftes Athmen nnd Rasselgeränsche [so macht z. B. Grisolle (l. c.) anf die Hänfigkeit derartiger Erscheinnngen anfnterksam, die sich viele Wochen lang lialten können], sondern selbst Bronchialathmen nnıl Bronchophonie hörbar sind, wälırend die Kranken (oder eigentlich nnn Gesnndea) sich absolnt wohl fühlen, uicht husten n.s.w. Ein Parallelgehen beider Erscheinungen (d. h. Bronchialathmen und Brouchophonie) findet nnn nicht inmer statl, wie ja das anch sonst nicht ohne Weiteres der Fall ist; schon Skoda (Ahlıandlung über Percussion nad Anscnltation) hat daranf hingerviesen.

Lebert (l. c.) giebt an, dass die Bronchophonie durchschuittlich früher auflört als das bronchiale Athmen - ich möchte grade das Gegentheil behanpten. Ich wässte mich keines Falles von Pnenmonie zn entsinaen, wo nicht Ungleich heiten in der hörbarea Stimme viel länger persistirt hätten, als Abweichungen des Athemgeränsches, und soviel ich weiss, immer liabe ich, wenn längst Bronchialathmen verschwnnden nnd wieder durch vesiculäres ersetzl war, die Stimme a nf der erkrankt gewesenen Seite deutlicher gehört als anf der gesnnden, mitunter Wochen lang. Zienıssen (l. c.) erwähnt, dass er Bronchialatlımen nud Bronchophonie bisweilen noch 2-3 Wochen nach der Entfiebernug forthestehend gefunden habe, erwälıt aber keine Unterschiede itn Verhalten beider; er betont jedoch die Wichtigkeit der Anscnltation der Stinıme ganz besonders.

v. C. Hueter. Grandriss der Chirargie. Leipzig 1884. Verlag von F. C. W. Vogel. Ref. Kolaczek.

Der Hueter'sche Groudriss der Chirnrgie in seiner zweiten, von Lossen sorgälttig durchgeséhenen Anflage lieğt vor uns. Das Bedürfniss einer nenen Anflage dieses Werkes nach Ablauf von kaum zwei Jahren seit dem ersten Erscheinen desselben beweist seine günstige Aufnalıme bei Aerzten nnd vielleicht anch Stndirenden. Jenen rnusste es willkommen sein, einen Grnudriss der Chirnrgie in ihrer nenesten Pliase aus der darch ihre meisterhafte Darstellnngsknnst rälımlichst bekannten Hand eines Hoter geliefert zn bekommen, wenn sie anch andererseits vorbereitet sein konnten, einen gewissen, hier and da hervortretenden Snbjectivismns mit in den Kanf zn nelımen. Die Studirenden wieder hattelı das lebhafte Bedürfniss, zunächst für den heschräıkten Schnläw'eck, nach einer Darstellngg der gesammten Chirurgie in noce, wie sie ihnen in keinem der gebränchlichen Lehrbücher geboten war. Einen Grondriss der Chirnrgrie nussten sie also vor Allen frendig begrüssen. Ob sich ilıre Hoffungen erfüllt haben? Das erscheint zweifelhaft, da dieser Grnndriss einen solchen Umfang erreicht hat, dass es ihnen nuter den 
gegebenen Verhältnissen kauın mörglich sein dürfte, während ihrer so kurzen academischen Lernzeit durch die Fülle des gebotenen Materials mit dem Erfolge sich hindurchzuarbeiten, dass sie das ganze Gebiet gleichmässig zu übersehen verı̈öchten und überall genügend Bescheid wüssten. Zudem mussten sie bald zur Ueberzeugung kommen, dass in vielen Dingen $\mathrm{zwischen}$ den in diesem Grundrisse niedergelegten und den ihnen von ihren Lehrern vorgetragenen Anschauungen eine missliche Differenz bestand. Diese hat ehen in dem schon erwähnten ausgeprägten Suhjectivisnus der Hueter'schen Lehrart ihreu Gruncl. Besonders machte sich derselbe im Gebiete der allgemeinen Chirurgie geltend.

Vielleicht aus dem zuletzt erwähnten Grunde begann Lossen bei der Vorbereitung der 2. Auflage des „Grundrisses“ mit dem speciellen Theile. Hier konnte nämlich die von der Pietät gegen den bürzlich Verstorbenen dictirte Schonung des von demselben Geschaffenen noch am Ehesteu geübt werden. L. begnügte sich deshalb mit einfach formellen Aenderuıgen, einer präciseren Darstellung der Diction, wo es nöthig war, der Zerlearung des etwas dickleibigen Bandes in drei Abtheilungen und dem Hinzufügen des auf dem Gebiete der speciellen Chirurgie in jüngster Vergangenheit hervorragend Geleisteten. Somit hat sich der Umfang dieses Haupthieils des Grundrisses sogar um etwas erweitert, also seinem Zwecke, Grundriss zu sein, wohl noch mehr entfremdet. Leider sind manche, von der Logik geforderte Aenderungen in der Anordnung des Stoffes unterblielien. So z. B. muss die Darstellung der Magenresection, vielleitht auch der Gastrostonie, aus dem Kapitel über Krankheiten der Speiseröhre entfernt werden. Dann dürfte es sich empfehlen, die Krankheiten des Mastdarms gesondert von denen des übrigen Darmcanals zu behandeln, da es sich eigenthümlich ausnimmt, die laparotomischen Eingriffe erst hinter der Behandlung der Hämorrhoidalknoten erörtert zu finden. Auch darf die Besprechung des Mastdarmkrebses und des Exstirpatio recti nicht zusammenhangslos an zwei verschiedenen, von einander weit alggelegenen Stellen stattfinden. Uebrigens dürfte auch des Mastdarmkrehses nicht gelegentlich der Abhandlung über Darmstricluren Erwähnung gethan werden; vielmelır müsste er in den Paragraphen, der von den Geschwülsten, hesonders des Rectum handelt, eine eingehende Berücksichtigung erfalıren. Die Hüt er'sche Theorie über die Aetiologie der halituellen Scoliose und des Klumpfusses tritt allzusehr in den Vordergrund, zumal sie kaum von irgend einer Seite her Anerkennung gefunden hat.

Durchgreifendere Aenderungen hat sich L. mit Recht veranlasst gesehen, im allg. Theile des Grundrisses vorzunehinen. Trotzdem er hier Manches, vornehmlich auf dem Gebiete der Bacteriologie neuerdings Geleistete linzugefügt hat, ist doch der Umfang dieses Theiles geringer geworden, weil andererseits Vieles fortgefallen und gedrängter zusammengefasst worden ist. Die materiellen Aenderungen springen besonders in den Kapiteln, die der Lehre von der Entzündung angehören, in die Augen. L. hat sich mit Recht entschlossen, zum Theil unter Berufung auf neueste experimentelle Thatsachen den exclusiv nycologischen Standpunkt Hueter's in der Entzündungsfrage zu verlassen und den Werth desselben als eines noch theoretischen deutlicher hervorzuheben. Doch hat in der Entzündungslehre nach ıneinem Dafürhalten die allgemeine Pathologie eine für einen Grundriss der Chirurgie noch zu weit gehende Aufnahme gefunden. Dalür alser dürfte die allgemeine $\mathrm{Ge}-$ schwulstlehre eine relativ zu knappe Berücksichtigung erfahren haben.

Nach alledem dürfte Hueter's Grundriss der Chirurgie richtiger in die Reihe der Handhücher eimzureihen sein. Als solches hat es seine grossen Vorıüge, den einheitlichen Guss, die klare und gefällige Form der Darstellıng, und wird in der Pflege Lossen's einen noch grösseren Werth erhalten, wenn seine bessernde Hand durch gewisse Rücksichten sich weniger heschränkt fühlen wird. 